\title{
Measurements of Higgs couplings to fermions and bosons at the LHC
}

\author{
Serhat Ördek* on behalf of the ATLAS and CMS Collaborations \\ Department of Physics and Astronomy, Uppsala University, \\ Lägerhyddsvägen 1, Uppsala, Sweden \\ E-mail: serhat.oerdek@cern.ch
}

With the full Run 2 pp collision dataset collected at $13 \mathrm{TeV}$, very detailed measurements of Higgs boson properties can be performed using its decays into bosons and fermions. At the same time, the search for Higgs-boson pair production can profit from the large integrated luminosity to provide more and more stringent limits. This document presents measurements of Higgs boson properties using decays into bosons and fermions and their combination, including production mode cross sections and simplified template cross sections, as well as their interpretations. Searches for non-resonant Higgs-boson pair production, as well as their combination, are also discussed.

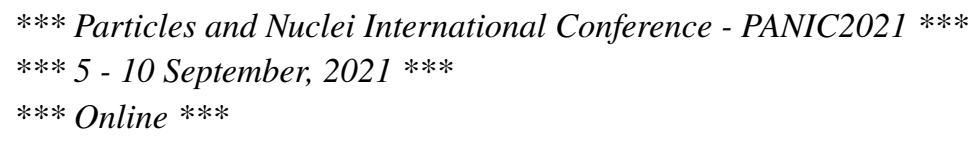

\footnotetext{
${ }^{*}$ Speaker
} 


\section{Introduction}

The Standard Model (SM) predicts the Higgs boson interactions to be described by:

$$
\mathcal{L}=-\frac{m_{f}}{v} \bar{f} f H+\frac{2 m_{W}^{2}}{v} W_{\mu} W^{\mu} H+\frac{m_{Z}^{2}}{v} Z_{\mu} Z^{\mu} H+\frac{m_{V}^{2}}{v^{2}} V_{\mu} V^{\mu} H^{2}+\frac{m_{H}^{2}}{2 v} H^{3}+\frac{m_{H}^{2}}{8 v^{2}} H^{4} .
$$

Here, $f$ represents the SM fermions, $v$ the vacuum expectation value of the Higgs field, and $V \in\{W, Z\}$. The Lagrangian $\mathcal{L}$ implies that Higgs bosons couple to all massive SM particles, and more strongly to particles with higher masses. Moreover, all couplings in the Lagrangian are purely CP-even. This report summarises the measurements conducted by the ATLAS [1] and CMS [2] collaborations to test these expectations using data collected at the LHC from 2015 to 2018.

\section{Cross Section Measurements}

A recent coupling measurement in an already observed decay channel is the ATLAS measurement in the $H \rightarrow \tau \tau$ final state [3]. To be able to measure the Higgs-boson production cross sections for the individual production mechanisms, the collected data are split into multiple signal regions (SRs), each with different relative contributions per production process. In each SR, the invariant di- $\tau$ mass distribution is used as input to a template likelihood fit. The resulting cross section values are shown in Figure 1 (left).
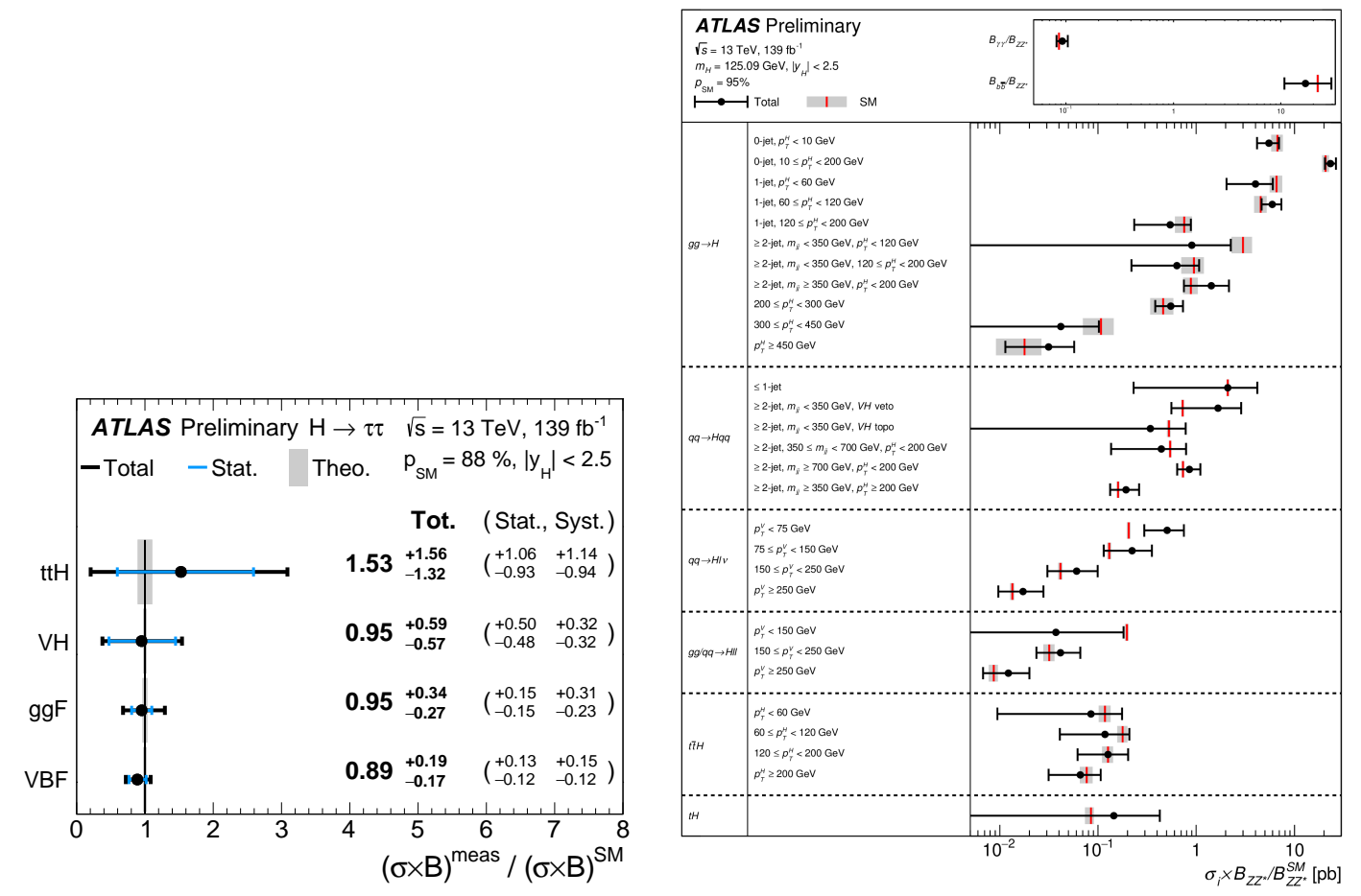

Figure 1: Left: Process-dependent Higgs-boson production cross sections obtained in the di- $\tau$ final state [3]. Right: Combined STXS measurement in the $H \rightarrow \gamma \gamma, H \rightarrow Z Z$ and $H \rightarrow b b$ final states [4]. 
To include kinematic information while minimising the impact of theoretical uncertainties, the simplified template cross section (STXS) framework was introduced. For this, each production process is split based on kinematic properties, such as the transverse momentum of the Higgs boson. A recent combined STXS measurement in ATLAS [4] is summarised in Figure 1 (right).

\section{Searches for Unobserved SM Couplings}

\section{1 $H \mu \mu$ Yukawa coupling}

Since the coupling of the Higgs boson to lighter particles is weaker, the $H \rightarrow \mu \mu$ branching ratio is relatively low, and the process has not yet been observed. Both the ATLAS and CMS experiments recently published analyses targeting $H \rightarrow \mu \mu$ decays [5, 6]. While ATLAS assumed only a Higgs-boson mass $m_{H}$ of $125.09 \mathrm{GeV}$, CMS conducted a scan in $m_{H}$. The observed local $p$-value in dependence of $m_{H}$ is shown in Figure 2 (left).
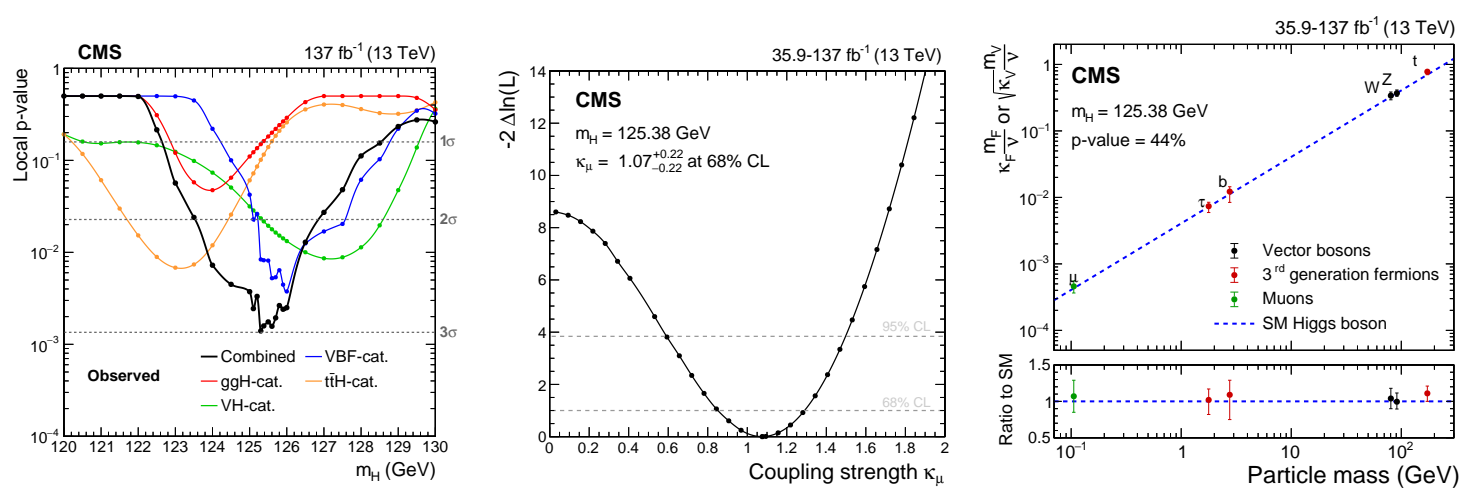

Figure 2: Left: Local $p$-value dependence on $m_{H}$ in the $H \rightarrow \mu \mu$ search. Centre: Measurement of the $H \mu \mu$ coupling modifier $\kappa_{\mu}$. Right: Higgs boson coupling strengths in dependence of the particle mass [5].

ATLAS observed (expected) a signal significance of 2.0 (1.7) $\sigma$. CMS obtained 3.0 (2.5) $\sigma$ for $m_{H}=125.38 \mathrm{GeV}$, reporting the first evidence of the $H \mu \mu$ coupling. CMS also measured the $H \mu \mu$ coupling strength modifier $\kappa_{\mu}=\Gamma_{\mu}^{\mathrm{Obs}} / \Gamma_{\mu}^{\mathrm{SM}}$ with the decay width $\Gamma_{\mu}$ of the Higgs boson into muons, as shown in Figure 2 (centre). Figure 2 (right) shows a comparison of coupling strengths over three orders of magnitude, confirming the SM predictions for all measured coupling strengths.

\subsection{Higgs Boson Pair Production}

The not yet observed Higgs-boson pair $(H H)$ production processes are sensitive to the modifiers of the trilinear self-coupling $\kappa_{\lambda}$ and the quartic vector-boson coupling $\kappa_{2 V}$. Gluon-gluon fusion (ggF) and vector-boson fusion (VBF) Feynman diagrams including these couplings are shown in Figure 3 (left). Studies on these couplings and the total cross section of $H H$ production have recently been conducted in the $b b \gamma \gamma$ final state. The observed (expected) cross section limits at $95 \%$ confidence level (C.L.) are 4.1 (5.5) times the cross section predicted by the SM by ATLAS [7] and 7.7 (5.2) times by CMS [9]. Constraints have also been placed on $\kappa_{\lambda}$, as shown in Figure 3 (centre). ATLAS has constrained the $H H$ production cross section to 4.7 (3.9) times the cross section 

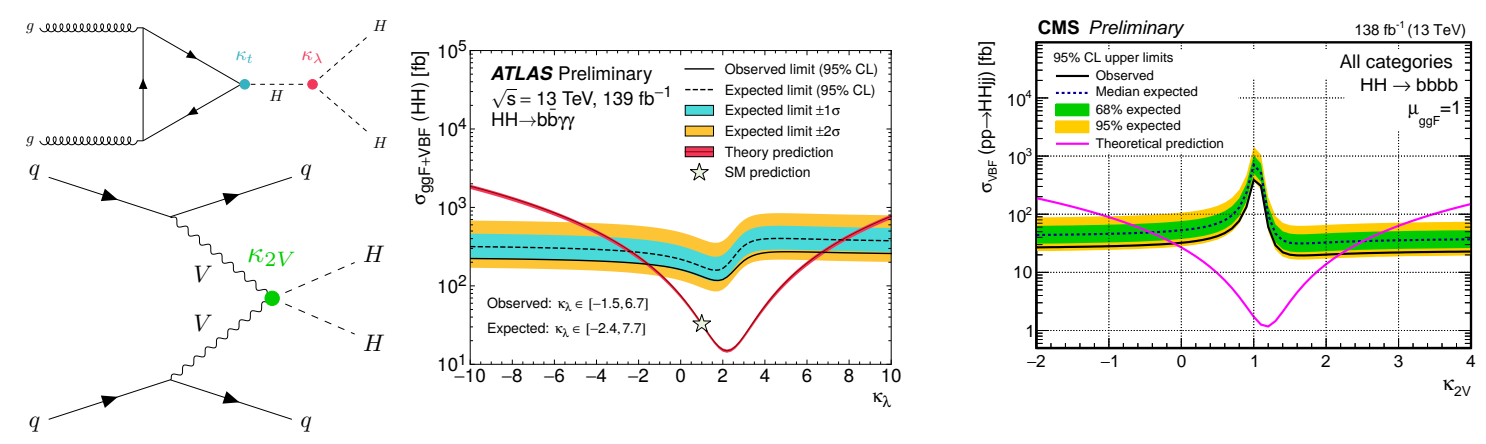

Figure 3: Left: Di-Higgs production Feynman diagrams showing the dependence on $\kappa_{\lambda}$ and $\kappa_{2} V$. Centre: $\mathrm{VBF}+\mathrm{ggF}$ cross section limit depending on $\kappa_{\lambda}$ [7]. Right: VBF cross section limit depending on $\kappa_{2 V}$ [8].

predicted by the SM using events in the $b b \tau \tau$ final state [10]. CMS has constrained it to 3.6 (7.3) times using the $4 b$ final state [8] and also placed constraints on $\kappa_{2} V$, as shown in Figure 3 (right).

\section{Searches for Non-SM Couplings}

The properties of Higgs boson Yukawa couplings to a fermion $f$ can be described by

$$
\mathcal{L}_{\text {Yukawa }}=-\frac{m_{f}}{v}\left(\bar{\psi}_{f} \kappa_{f}\left[\cos (\alpha)+\mathrm{i} \sin (\alpha) \gamma^{5}\right] \psi_{f}\right) H
$$

with the CP-mixing angle $\alpha$. Using $t t H$ events and the $H \rightarrow \gamma \gamma$ decay, the CP-odd and CP-even contributions to the top-Yukawa coupling were measured [11], as shown in Figure 4 (left).
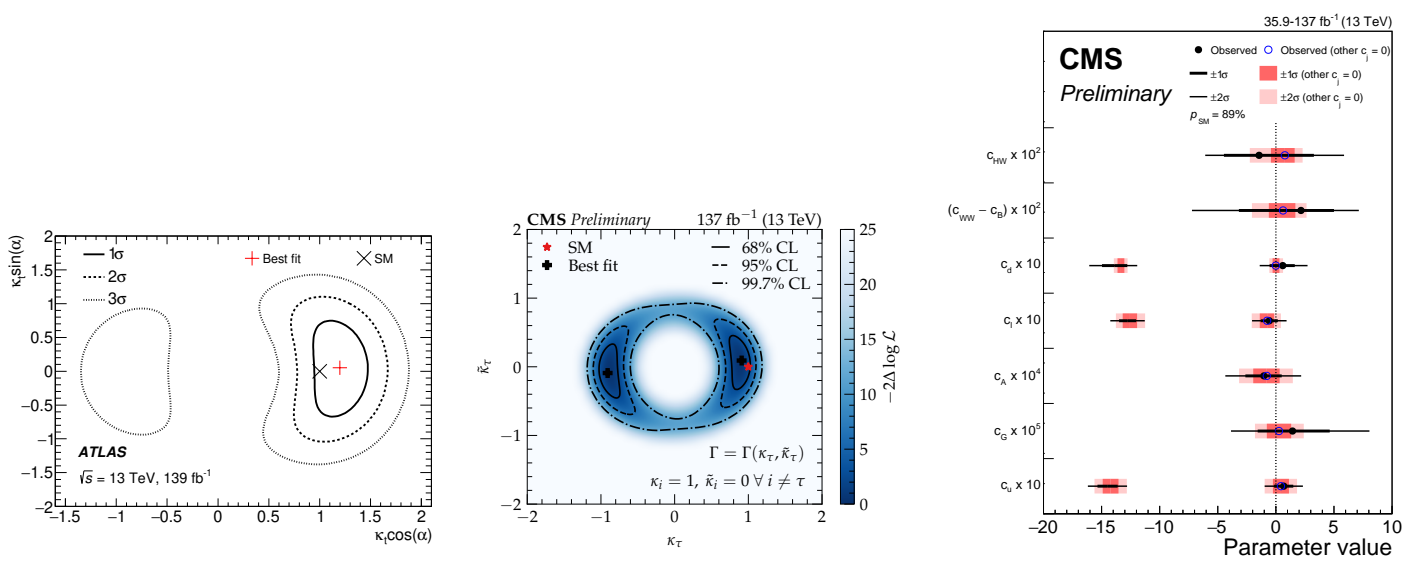

Figure 4: Left: Measurement of CP-odd and CP-even contribution to the top-Yukawa coupling [11]. Centre: Measurement of CP-odd $\left(\tilde{\kappa}_{\tau}\right)$ and CP-even $\left(\kappa_{\tau}\right)$ contribution to the $\tau$-Yukawa coupling [12]. Right: Measurement of Wilson coefficients of CP-even EFT operators [13].

Figure 4 (centre) shows a similar measurement of the CP properties of the $H \tau \tau$ coupling, measured in the di- $\tau$ final state [12]. Both measurements support the SM prediction of purely CPeven couplings. To test further couplings not predicted by the SM, effective field theories (EFTs) 
can be employed. In EFTs, dimensionless Wilson coefficients $c_{i}$ quantify the strength of additional couplings $i$. A combined measurement [13] is summarised in Figure 4 (right), showing agreement of all investigated $c_{i}$ with zero. Hence, no sign of additional couplings was found.

\section{References}

[1] ATLAS Collaboration, The ATLAS Experiment at the CERN Large Hadron Collider, JINST 3 (2008) S08003.

[2] CMS Collaboration, The CMS experiment at the CERN LHC, JINST 3 (2008) S08004.

[3] ATLAS Collaboration, Measurements of Higgs boson production cross-sections in the $H \rightarrow \tau \tau$ decay channel in pp collisions at $\sqrt{s}=13 \mathrm{TeV}$ with the ATLAS detector, ATLAS-CONF-2021-044.

[4] ATLAS Collaboration, A combination of measurements of Higgs boson production and decay using up to $139 \mathrm{fb}^{-1}$ of proton-proton collision data at $\sqrt{\mathrm{s}}=13 \mathrm{TeV}$ collected with the ATLAS experiment, ATLAS-CONF-2020-027.

[5] CMS Collaboration, Evidence for Higgs boson decay to a pair of muons, JHEP 01 (2021) 148.

[6] ATLAS Collaboration, A search for the dimuon decay of the Standard Model Higgs boson with the ATLAS detector, Phys. Lett. B 812 (2021) 135980.

[7] ATLAS Collaboration, Search for Higgs boson pair production in the final state with two bottom quarks and two photons in pp collisions at $\sqrt{s}=13 \mathrm{TeV}$ with the ATLAS detector, ATLAS-CONF-2021-016.

[8] CMS Collaboration, Search for Higgs boson pair production in the four b quark final state, CMS-PAS-HIG-20-005.

[9] CMS Collaboration, Search for nonresonant Higgs boson pair production in final states with two bottom quarks and two photons in proton-proton collisions at $\sqrt{s}=13 \mathrm{TeV}, \mathrm{JHEP} 03$ (2021) 257.

[10] ATLAS Collaboration, Search for resonant and non-resonant Higgs boson pair production in the $b \bar{b} \tau^{+} \tau^{-}$decay channel using $13 \mathrm{TeV} p \mathrm{p}$ collision data from the ATLAS detector, ATLAS-CONF-2021-030.

[11] ATLAS Collaboration, CP Properties of Higgs Boson Interactions with Top Quarks in the $t \bar{t} H$ and $t H$ Processes Using $H \rightarrow \gamma \gamma$ with the ATLAS Detector, Phys. Rev. Lett. 125 (2020) 061802.

[12] CMS Collaboration, Analysis of the CP structure of the Yukawa coupling between the Higgs boson and $\tau$ leptons in proton-proton collisions at $\sqrt{s}=13 \mathrm{TeV}$, CMS-PAS-HIG-20-006.

[13] CMS Collaboration, Combined Higgs boson production and decay measurements with up to $137 \mathrm{fb}^{-1}$ of proton-proton collision data at $\sqrt{s}=13 \mathrm{TeV}$, CMS-PAS-HIG-19-005. 